\title{
Plastic Instability Mechanisms in Bimetallic Nanolayered Composites
}

\author{
S.J. Zheng ${ }^{\mathrm{a},{ }^{*}, \text { J. Wang }^{\mathrm{b}} \text {, J.S. Carpenter }}{ }^{\mathrm{c}}$, W.M. Mook ${ }^{\mathrm{a}, \# \text {, P.O. Dickerson }}{ }^{\mathrm{c}}$, N.A. Mara ${ }^{\mathrm{a}, \mathrm{c}}$,
} I.J. Beyerlein ${ }^{\mathrm{d}}$

${ }^{a}$ Center for Integrated Nanotechnologies, MPA-CINT, Los Alamos National Laboratory, Los Alamos, NM 87545, USA.

${ }^{\mathrm{b}}$ Materials Science and Technology Division, MST-8, Los Alamos National Laboratory, Los Alamos, NM 87545, USA.

${ }^{c}$ Materials Science and Technology Division, MST-6, Los Alamos National Laboratory, Los Alamos, NM 87545, USA.

d Theoretical Division, T-3, Los Alamos National Laboratory, Los Alamos, NM 87545, USA.

\# Current address: Center for Integrated Nanotechnologies, Sandia National Laboratories, Albuquerque, NM 87185, USA.

*Corresponding author, email address: sjzheng@lanl.gov, or sjzhengsj@gmail.com (SJ Zheng).

\begin{abstract}
Strain localization is a common deformation-induced instability in many metallic metals. How it happens is related to both microstructure and the way in which plasticity is mediated prior to localization. Both aspects can fundamentally change in a face-centred cubic metal when it becomes nanostructured; the propensity for deformation twinning increases and the behaviour is dominated by dislocation-interface interactions. Here we carry out a TEM investigation to elucidate the collaborative role of deformation twinning and dislocation transmission on the onset of strain localization in nanolayered composites. Two material systems are examined, $\mathrm{Cu}-\mathrm{Ag}$ and $\mathrm{Cu}-\mathrm{Nb}$, and for each system, two interface structures are examined, one prone to dislocation transmission and the other not. We show that dislocation transmission favours crystallographic band formation, whereas dislocations that do not transmit cause interface tilting and are associated with (non-crystallographic) shear band formation.
\end{abstract}

Keywords: interfaces, twinning, shear bands, nanolayered composites, TEM 


\section{Introduction}

Two-phase nanolayered composites possess many desirable qualities: high strength, high hardness, thermal stability, radiation damage tolerance, and shock resistance [1-6]. Another property critical in the design of nanomaterials for structural applications is macroscopic ductility. Many studies have shown that ductility in such layered materials is limited by the eventual formation of localized bands of intense plastic strain [7-10]. In efforts to postpone such instabilities and enhance ductility, it is desirable to understand the microstructural features that are responsible for their inception.

Towards this end, prior work on plastic instabilities in nanolayered composites focused on $\mathrm{Cu}-\mathrm{Nb}$ thin films produced by physical vapor deposition (PVD) [7], which possess planar $\mathrm{Cu}-$ $\mathrm{Nb}$ interfaces with a Kurdjumov-Sachs orientation relationship [11, 12]. Using micropillar compression, it was found that within the shear band that had run across the pillar, the layers had rotated to an orientation favourable for interfacial sliding [7]. The particular interface in PVD Cu-Nb is known to slide easily [13]. This response shares some key aspects with shear bands in conventional composites in that the shear bands were 1) non-crystallographic and 2) promoted when deformation is applied parallel to the layers.

When the same test was performed on a $\mathrm{Cu}-\mathrm{Nb}$ nanolayered composite fabricated by accumulative roll bonding (ARB) possessing crystallographically different interfaces more resistant to interfacial sliding, the mode of failure was described as being less localized, involving many distributed shear bands [8]. Clearly the structure of the interface and interactions the interface has with slip dislocations had played a role in how these two nanomaterials softened under deformation. However, the mechanism or the critical event that leads to banding in the first place has yet to be clarified or analyzed in depth. If the interface structure were to change, how would the critical event and the plastic instability it creates change?

The focus of this work lies in uncovering the mechanisms that lead to strain localization in the form of a band in nanolayered composites. Different nanolayered structures are studied and so the generalized definition of plastic instability we adopt is intended to present the possibility that a twin band, slip band, or non-crystallographic, shear band could form.

Because of the fine nanostructure, a different perspective than those developed for coarserlayered materials may be required. For strain localizations in coarse-layered composites, 
interpretation and modelling have found that individual dislocations or groups of dislocations are not primarily responsible for the critical shear event [14-18]. These assumptions are valid as the layers are polycrystalline and contain many grain boundaries. Dislocation tangles form in the grains and dislocation pile-ups can form at the grain boundaries and interfaces. These defect structures can generate strain concentrations that can potentially trigger instability. In fine nanolayered composites, however, in which the interface spacing is on the order of a few tens of nanometers or less, there is no room within the crystals to accommodate dislocation pile-ups or substructure. It then becomes important to consider the interactions of individual dislocations with the interface.

There are several types of defect-interface interactions. Upon impinging on an interface, a dislocation can be transmitted or absorbed and stored [13, 19-21]. The outcome of this interaction depends on the type of dislocation, whether a slip or twinning dislocation, and the type of interface, whether it resists transmission or not. With this in mind, we design a set of nanomechanical deformation experiments to elucidate the role of dislocation-interface interactions on the onset of strain localization. To explore dependencies on the type of dislocation involved, we choose two bi-phase composite material systems, one in which one phase readily twins $(\mathrm{Cu}-\mathrm{Ag})$ and another in which both phases predominantly slip $(\mathrm{Cu}-\mathrm{Nb})$. To further study the role of interface transmission, we choose systems that contain interface structures in which a dislocation can be expected to either transmit across or become stored in the interface. Our findings elucidate that whether twinning or slip dislocations mediate plasticity, dislocation interface transmission leads to crystallographic band formation and resistance to transmission leads to (non-crystallographic) shear banding.

\section{Experimental}

A nanolayered $\mathrm{Cu}-\mathrm{Ag}$ composite with a eutectic composition (40at.\% $\mathrm{Cu}$ ) was fabricated via a flux-melting technique [22]. The as-prepared material is a rod with a diameter of $8 \mathrm{~mm}$ and a height of about $5 \mathrm{~cm}$. A section of the rod was subjected to $11 \%$ compression in height, and a bar $(3 \mathrm{~cm} \times 8 \mathrm{~mm} \times 1.5 \mathrm{~mm}$, length $\times$ width $\times$ thickness $)$ was cut off from the as-prepared rod and then was rolled down $89 \%$ in thickness at room temperature.

A PVD Cu-Nb composite was deposited on Silicon substrates, and an ARB Cu-Nb composite was fabricated via repeated rolling, cutting, stacking, and rolling, at room temperature. The PVD $\mathrm{Cu}-\mathrm{Nb}$ and $\mathrm{ARB} \mathrm{Cu}-\mathrm{Nb}$ micropillar compressions were carried out on a Hysitron ${ }^{\mathrm{TM}}$ nanoindenter and an Agilent NanoXP nanomechanical tester, respectively. The testing was 
executed in displacement control with the Continuous Stiffness Mode disabled at an initial strain rate of $1 \times 10^{-4} \mathrm{~s}^{-1}$. The details about material fabrication and pillar testing can be found in our previous work [7, 23].

Microstructures were investigated by transmission electron microscopy (TEM). TEM samples except for that cut from the deformed micropillars by FIB, were prepared by the conventional method including mechanical grinding, polishing, and ion milling. TEM observation was performed on an image-corrected Titan 80-300 (FEI).

\section{Results and Discussion}

\subsection{Cu-Ag Composite}

We first consider the two-phase $\mathrm{Cu}-\mathrm{Ag}$ system. Due to its low stacking fault energy of 26 $\mathrm{mJ} / \mathrm{m}^{2}$ [24], Ag is prone to deformation twinning, especially for finely sized crystals, such as the case in this work and under large deformation $[25,26]$. In contrast, $\mathrm{Cu}$ has a medium stacking fault energy of $45 \mathrm{~mJ} / \mathrm{m}^{2}$ [24], which means twinning in $\mathrm{Cu}$ is not as easy as in $\mathrm{Ag}$ and usually requires low temperature or high strain rates $[27,28]$. However, the propensity for twinning at room temperature in $\mathrm{Cu}$ tends to increase as the crystal size reduces from the coarse-scale to the nanoscale $(<100 \mathrm{~nm})[3,29-32]$.

The $\mathrm{Cu}-\mathrm{Ag}$ nanocomposite is comprised of colonies (Fig. 1a), and each colony consists of $\mathrm{Cu}-\mathrm{Ag}$ nanolamellae with average individual layer thicknesses of $30(\mathrm{Cu})$ and $65(\mathrm{Ag}) \mathrm{nm}$ (Fig. 1b), respectively [33]. As demonstrated by the <110> composited electron diffraction patterns in Fig. 1b, the $\mathrm{Cu}-\mathrm{Ag}$ orientation relationships in the present samples can be characterized as either cube-on-cube (Ag green pattern-Cu red pattern, Ag yellow pattern- $\mathrm{Cu}$ white pattern) or hetero-twin (Ag green pattern-Cu white pattern, $\mathrm{Ag}$ yellow pattern-Cu red pattern), both with $\{111\}$ interface planes, which are consistent with literature [33, 34]. These two interface structures co-exist, but the hetero-twin interfaces make up the majority $[34,35]$.

We used high resolution TEM (HRTEM) to analyze and compare the atomic structures of these types of interface structures. Figure 2a displays a HRTEM image of a typical $\{111\} \mathrm{Cu} / /\{111\} \mathrm{Ag}$ interface with cube-on-cube orientation relationship in the as-cast sample. The cube-on-cube orientation relationship is demonstrated in Fig. 2c. Due to the $\mathrm{Cu}-\mathrm{Ag}$ lattice mismatch, misfit dislocations, as well as atomic steps indicated with a red arrow, are present at the interface. Figure $2 b$ shows a HRTEM image of the $\{111\} \mathrm{Cu} / /\{111\} \mathrm{Ag}$ hetero- 
twin interface possessing similar misfit dislocations and atomic steps as the cube-on-cube $\mathrm{Cu}$ Ag interface since they have the same lattice mismatch. The misfit dislocations and atomic steps facilitate dislocation nucleation $[13,36]$. However, different from the cube-on-cube $\mathrm{Cu}$ Ag interface, the lattice and hence slip systems across the hetero-twin interface have a mirror symmetry orientation relationship, as illustrated in Fig. 2d.

As a measure of comparing the transmissibility of two interfaces for the $\mathrm{Cu}-\mathrm{Ag}$ system, as well as the $\mathrm{Cu}-\mathrm{Nb}$ system analyzed later, we consider using a geometric factor denoted as $\chi$ that qualifies the alignment of two slip systems across an interface. To develop this measure, we begin with the model proposed by Werner and Prantl [37] $G=\left\langle\cos \left(\frac{\pi}{2} \frac{\Psi^{\alpha \beta}}{\Psi_{\mathrm{c}}}\right)\right\rangle\left\langle\cos \left(\frac{\pi}{2} \frac{\theta^{\alpha \beta}}{\theta_{\mathrm{c}}}\right)\right\rangle$, where $\alpha$ and $\beta$ represent the two grains (phases) composing the boundary (interface), $\Psi_{\alpha \beta}$ is the angle between the slip directions (Burgers vector of the slip dislocations) and $\theta^{\alpha \beta}$ is the angle between the intersection lines formed by the two slip planes and the boundary (interface) plane. The brackets $<>$ mean that when either $\Psi_{\alpha \beta}$ or $\theta_{\alpha \beta}$ exceeds their assigned limit $\Psi_{c}\left(45^{\circ}\right)$ or $\theta_{c}\left(15^{\circ}\right)$, this quantity is zero. To additionally consider the driving force on the incoming and outgoing systems, we multiply this factor by their Schmid factors $m_{\alpha}$ and $m_{\beta}$, which gives $G=\left\langle\cos \left(\frac{\pi}{2} \frac{\Psi^{\alpha \beta}}{\Psi_{c}}\right)\right\rangle\left\langle\cos \left(\frac{\pi}{2} \frac{\theta^{\alpha \beta}}{\theta_{c}}\right)\right\rangle\left[m_{\alpha}\right]_{\xi}$. The []$\xi$ brackets indicate that the Burgers vector of the slip dislocations are defined such that the dislocation transmitting from slip system $\alpha$ to system $\beta$ has the same line sense $\xi$ [38]. Finally, we define the geometric efficiency factor for interface transmission as $\chi=\mathrm{G}_{\mathrm{max}} / \mathrm{G}_{0}$, where $G_{\max }$ is the transmission tendency of the most geometrically favourable pair of slip systems for a given interface and $\mathrm{G}_{0}=0.25$ is simply the largest possible value for $\mathrm{G}$.

Another property of the interface character that greatly impacts transmissibility is the interface structure [38]. Its influence is not as straightforward to qualify as the geometric aspect. Nonetheless, based on some MD simulations, correlations have been drawn between low interfacial shear resistance with the difficulty of dislocation transmission [39]. The reasoning is that incoming dislocations tend to become absorbed and spread their cores within the interface plane when the interfacial shear strength is low. This response hinders their transmission to the adjoining crystal. We will revisit this aspect for each of the interfaces studied here.

As one means of promoting strain localization, a $1.2 \mathrm{~cm}$ high section of the cast $\mathrm{Cu}-\mathrm{Ag}$ rod was subjected to $11 \%$ uniaxial compression. Figure 3 a shows a TEM image of the 
compressed $\mathrm{Cu}-\mathrm{Ag}$ composite. Substantial twins are present in the Ag layer. Of particular interest, the twins have crossed the $\mathrm{Cu}-\mathrm{Ag}$ interfaces, as well as the whole $\mathrm{Cu}$ layer. Within this cross-interface twin band, the pair of twinning systems on either side of the interfaces are well aligned and the cube-on-cube orientation relation is maintained within the band. This localized band can clearly be classified as crystallographic and not a shear band.

These trans-interface twin bands are associated with the cube-on-cube $\{111\} \mathrm{Cu} / /\{111\} \mathrm{Ag}$ $\mathrm{Cu}-\mathrm{Ag}$ interface and develop because of interface facilitated (twinning) dislocation transmission. Using the slip system nomenclature in Fig. 2c, for example, we consider Ag twinning partials $\alpha \mathrm{B}_{\mathrm{Ag}}$ and $\alpha \mathrm{C}_{\mathrm{Ag}}$, which have identical, favourable Schmid factors for the cystallographic orientation of this crystal with respect to the loading. As illustrated in Fig. 3b$3 \mathrm{c}$, under the prescribed loading, the cube-on-cube $\mathrm{Cu}-\mathrm{Ag}$ orientation relationship provides excellent alignment between an active $\mathrm{Ag}$ twin system, such as $\alpha \mathrm{B}_{\mathrm{Ag}}$, and the $\mathrm{Cu}$ twin system, $\alpha{ }^{\prime} \mathrm{B}_{\mathrm{Cu}}$, across the interface with a high $\chi$ value of 0.80 , so $\alpha \mathrm{B}_{\mathrm{Ag}}$ partial can transmit across the interface and transform into an active $\alpha^{\prime} \mathrm{B}^{\prime} \mathrm{Cu}$ partial. In fact for this interface, all twin systems on active twin planes (not just $\alpha \mathrm{B}_{\mathrm{Ag}}$ ), inclined to the interface, are well aligned with another twin system across the interface, raising the possibility of trans-interface twin band formation.

This trans-interface twin band can be viewed as an instability as it locally changes the state of the interface. Geometrical considerations alone can prove that a trans-interface twin band will not only reorient both $\mathrm{Cu}$ and $\mathrm{Ag}$ crystals to twin orientation but also reorient the interface from (111) to either (001) or other $\{111\}$, which depends on the Burgers vector of the twinning partials [40]. As confirmation, Fig. 3d shows that after twinning the $\mathrm{Cu}-\mathrm{Ag}$ interface indicated by dashed line has reoriented from (111) Cu//(111)Ag significantly. Moreover, the difference between the Burgers vector of the twinning partials in $\mathrm{Cu}$ and $\mathrm{Ag}(0.1476 \mathrm{~nm}$ in $\mathrm{Cu}$ and $0.1670 \mathrm{~nm}$ in $\mathrm{Ag}$ ) leaves residual dislocations in the interface that can accumulate in the interface. Storage of these relatively tiny residual defects can leave the interface in an unstable state. Prior experimental studies have shown that in such events the interface can respond by emitting fine secondary twins [33] or restructuring [40, 41].

To study another form of instability, the $\mathrm{Cu}-\mathrm{Ag}$ composite was subjected to $89 \%$ rolling deformation at room temperature. The deformation was sufficient to cause shear bands to form as shown in Fig. 4a, which is consistent with prior studies on rolled $\mathrm{Cu}-\mathrm{Ag}$ eutectic [9]. 
Figure 4 a displays a typical shear band. In the central core zone of the shear band, the layered morphology is preserved, the layer thickness has become finer, and the layers have rotated relative to those in the matrix. As indicated by the inserted corresponding diffraction patterns, in both the matrix and the shear band, the interfaces have deviated from the $\{111\}$ planes of $\mathrm{Cu}$ and $\mathrm{Ag}$. Furthermore, the shear band structure is not aligned with any crystallographic plane (non-crystallographic banding).

Consistent with a prior electron backscattering diffraction (EBSD) study on rolled $\mathrm{Cu}-\mathrm{Ag}$ [9], our TEM analysis indicates that the shear band is associated with the hetero-twin interface. In addition, TEM analysis reveals a mechanism that links the shear bands with the inability of Ag twins to transmit across this interface. As shown in Fig. $4 \mathrm{~b}$, which was taken from a matrix region near the shear band in Fig. 4a, deformation twins have formed in the Ag layers beside a thin $\mathrm{Cu}$ layer. In Fig. 4c, a HRTEM image is provided showing a detailed view of the interface shown in Fig. 4b. Here, the hetero-twin orientation of the interface can still be distinguished. As shown, the twins in Ag cannot transmit across the interface. For this interface and plane strain compression, the highest value of $\chi$ is 0.16 , indicating that geometrically this interface is not readily transmissible.

Because the twinning dislocations do not transmit, they become absorbed into the interface, as illustrated in Fig. 4d. As more accumulate with more twin-interface interactions, they can cause the interface to tilt. These stored dislocations will have both a planar $\perp$ and non-planar dislocation + component, the latter being the less mobile of the two (see Fig. 4d). Accumulation of the + dislocations can cause interface tilting, as demonstrated in Fig.4e. Evident local interface tilting, which is about $18^{\circ}$, is illustrated in Fig. $4 \mathrm{~b}$.

The inability of twinning dislocations to transmit across this interface could have triggered the shear band. We speculate that the $\mathrm{Cu}$ crystal accommodates interface tilting by activating slip parallel to the interface plane. This local change in slip activity could favour large crystallographic rotations and lead to shear banding.

To conclude this section on $\mathrm{Cu}-\mathrm{Ag}$ nanolayered composites, we find that the transmissibility of the interface profoundly changes the mode of strain localization. The material sections with a predominantly cube-on-cube orientation relationship were unstable under deformation due to trans-interface twin bands, since this interface permits the twinning dislocations readily activated in $\mathrm{Ag}$ to easily transmit across it into $\mathrm{Cu}$. In contrast, the material regions with a predominant hetero-twin orientation relationship are resistant to twin transmission. 
These areas become eventually unstable since Ag twinning dislocations that run into the interface cause it to tilt crystallographically. It is proposed that interface tilting can eventually trigger a) layer parallel slip, b) large crystal reorientations, and c) shear banding.

\subsection{Cu-Nb Composites}

$\mathrm{Cu}-\mathrm{Nb}$ is the second composite system we examine. Unlike the $\mathrm{Cu}-\mathrm{Ag}$ system, both metals deform primarily via slip and not twinning, even for the nano-sized crystals. To explore the effects of dislocation interface transmission, it is necessary to change the $\mathrm{Cu}-\mathrm{Nb}$ interface structure via sample fabrication. It is well established that PVD $\mathrm{Cu}-\mathrm{Nb}$ films possess a predominant $\{111\} \mathrm{Cu}-\{110\} \mathrm{Nb}$ Kurdjumov-Sachs (K-S) interface [42]. It was discovered recently that ARB process produces nanolayered sheets with a predominant $\{112\} \mathrm{Cu} \|\{112\} \mathrm{Nb}$ interface also with a K-S orientation relationship $\{111\}<110>\mathrm{Cu} \|\{110\}<111>\mathrm{Nb}[11,43]$. Both types of $\mathrm{Cu}-\mathrm{Nb}$ samples are tested here: the $\mathrm{Cu}-\mathrm{Nb}$ nanolayered composite with individual layer thickness $\mathrm{h}$ of $40 \mathrm{~nm}$ and the ARB Cu$\mathrm{Nb}$ nanolayered composite with an $\mathrm{h}=18 \mathrm{~nm}[23]$.

Figures 5a and 5b display typical TEM images of the as-deposited PVD and as-fabricated $\mathrm{ARB} \mathrm{Cu}-\mathrm{Nb}$ composites, respectively. As shown, in both composites, the $\mathrm{Cu}-\mathrm{Nb}$ interfaces are flat and sharp at low magnification, and both the $\mathrm{Cu}$ and $\mathrm{Nb}$ grains are broad in the directions parallel to the interfaces and a single grain spans the entire layer normal to the interfaces, especially in the $\mathrm{ARB} \mathrm{Cu}-\mathrm{Nb}$ [2]. However, these two interfaces differ significantly in their atomic scale structure. Figures $5 \mathrm{c}$ and $5 \mathrm{e}$ present respectively a HRTEM image of the $\mathrm{K}-\mathrm{S} \mathrm{Cu}-\mathrm{Nb}$ interface in the $\mathrm{Cu}-\mathrm{Nb}$ PVD foil and a geometric schematic diagram of the interface. The interface formed by $(111)_{\mathrm{Cu}}$ planes and $(01 \overline{1})_{\mathrm{Nb}}$ planes, which correspond to preferred slip planes in fcc and bcc metals, respectively, is atomically flat and contains misfit dislocations due to the difference of lattice parameters. Figure $5 \mathrm{~d}$ shows a typical HRTEM image of the ARB $\mathrm{Cu}-\mathrm{Nb}$ interface, which is comprised of a regular array of atomic-scale facets, which has been shown via MD simulations to be an intrinsic structural feature of this interface [30]. Thus, these facets were not created by dislocation-defect intersections. Moreover, similar to the PVD $\mathrm{Cu}-\mathrm{Nb}$ interface, the $\mathrm{ARB} \mathrm{Cu}-\mathrm{Nb}$ interface also presents misfit dislocations that can facilitate dislocation nucleation [21]. To show the interface geometry clearly, a schematic diagram of the ARB $\mathrm{Cu}-\mathrm{Nb}$ interface is shown in Fig. $5 f$. 
We also anticipate that these two interfaces differ significantly in their resistance to dislocation transmission. The PVD interface is expected to be the more resistant one for two main reasons. First, among the slip systems non-planar to the interface, only those on the $(\overline{1} \overline{1} 1)_{\mathrm{Cu}}$ and $(10 \overline{1})_{\mathrm{Nb}}$ in Figs. 5c and 5e are well-aligned, sharing the intersection line BC with the interface. The most well aligned slip systems $\mathrm{DB}_{\mathrm{Cu}}(1 / 2[\overline{1} 0 \overline{1}])-\mathrm{CF}_{\mathrm{Nb}}(1 / 2[1 \overline{1} 1])$ achieve a $\chi$ value of only 0.51. Secondly, according to previous MD simulations [44], this interface has a relatively low interfacial shear resistance. As the dislocation approaches this interface, its stress field can cause the interface to slide, which in turn attracts and traps the impinging dislocation, and once absorbed causes its core to spread within the interface, making it difficult to transmit to the other crystal. In experimental hardness tests, prevalent dislocation interface transmission is believed to manifest as a peak in the hardness vs. layer thickness curve [45]. Hardness tests indicate that dislocation transmission would not become dominant until the layer thickness decreased below $5 \mathrm{~nm}[46,47]$. Therefore for the $\mathrm{h}=40 \mathrm{~nm} \mathrm{Cu}-\mathrm{Nb}$ PVD foil with K-S interfaces tested in this work, the layer thickness is much larger than what is associated with prevalent dislocation transmission.

The ARB interface, on the other hand, is expected to more easily undergo dislocation transmission. First, with respect to geometrically efficient slip transmission pathways, the ARB interface is similar or slightly favoured over the PVD interface. As illustrated in Fig. 5f, the most aligned slip systems are $\mathrm{AB}_{\mathrm{Cu}}(1 / 2[01 \overline{1}])-\mathrm{CF}_{\mathrm{Nb}}(1 / 2[1 \overline{1} 1])$, which has a $\chi$ of 0.58 under a uniaxial compression state. In the particular HRTEM image Fig. 5d, the (111)Cu plane and the $(10 \overline{1}) \mathrm{Nb}$ plane are oriented by $22^{\circ}$ and $30^{\circ}$, respectively, about the $\mathrm{x}$-axis and from the $\mathrm{y}$ axis. As indicated by our previous texture study [23], these angles for $(111)_{\mathrm{Cu}}$ and $(10 \overline{1})_{\mathrm{Nb}}$ are narrowly distributed. Second, MD simulations find that the ARB interface has a much higher interface shear resistance than that of the PVD K-S interface due to its faceted and non-slip plane interface features $[48,49]$. Consequently, a dislocation that has been absorbed by the ARB interface would tend not to spread as much within the interface planes and be more apt to transmit to the other side. In support of this notion, in MD simulation, the stresses associated with dislocation interface transmission events across the ARB interface are lower than those across the PVD interface [20, 50]. As further support, comparison of hardness measurements between the ARB and PVD Cu-Nb composites show that peak hardness in the ARB composite is achieved around $20 \mathrm{~nm}$, whereas recall for the PVD composite it is attained at $5 \mathrm{~nm}[8,47]$. Thus, the strength of the ARB nanolayered composite tested here, 
which has a layer thickness $\mathrm{h}=18 \mathrm{~nm}$, is associated with ease of dislocation transmission [47].

To invoke a plastic instability, both materials were deformed in micro-pillar compression applied normal to the interfaces. These nanomechanical tests have been performed previously in $[7,8]$ and the procedures used here are the same.

For the PVD sample, a compression of 0.36 true strain was conducted on a micropillar. Figure 6a shows a TEM image of the deformed pillar. A shear band has gone through the sample. Layers are still continuous across the shear band, but have become thinner than their counterparts in the matrix. These observations are the same as those in prior work [7].

For the shear band in Fig. 6a, the K-S orientation relationship is preserved but altogether the $\mathrm{Cu}$ and $\mathrm{Nb}$ crystallographic orientations have reoriented by $28.5^{\circ}$, as indicated by a comparison between the insets corresponding to electron diffraction patterns of the shear band and the matrix, respectively. The morphology of the $\mathrm{Cu}-\mathrm{Nb}$ interface has only rotated by $17.5^{\circ}$. The difference signifies that the crystallographic interface planes have tilted $11^{\circ}$ and no longer corresponds to the $\{111\} \mathrm{Cu} \|\{110\} \mathrm{Nb}$ planes. Thus, like the hetero-twin $\mathrm{Cu}-\mathrm{Ag}$ interface, a shear band is associated with an interface that is resistant to dislocation transmission. Moreover, as before with the hetero-twin $\mathrm{Cu}-\mathrm{Ag}$ interface, we find the inability of the dislocations to transmit is associated with a crystallographic tilt in the interface plane. As illustrated in Figs. 6b-6c, when incoming dislocations become stored in the interface, the interface tilts due to the accumulation of the non-planar component $\vdash$ of the dislocations that are absorbed and not transmitted. The original layers lie normal to the loading direction and hence slip systems whose glide planes are parallel to the interface are not favored. However, the tilted interface plane can favour activation of slip systems parallel to the interface and eventual shear banding.

For the $\mathrm{Cu}-\mathrm{Nb}$ ARB sample, micropillar compression was carried out to 0.04 true strain. During FIB-TEM sample preparation, we used scanning electron microscopy (SEM) equipped in the FIB system to investigate the deformed microstructure. Figures $7 \mathrm{a}$ and $7 \mathrm{~b}$ show the micropillar before and after the compression, respectively. The arrow in Fig. $7 \mathrm{~b}$ indicates a localized band within the deformed pillar. The corresponding SEM image shown in Fig. 7c takes a closer look. In the SEM image, this band is observed to run across the pillar. As in the PVD shear band (Fig. 6a), the layers across this band boundary are continuous. However unlike the PVD case, the band angle $\beta$ is only $\sim 25^{\circ}$. This angle is smaller than that 
in the compressed PVD Cu-Nb pillar (Fig. 6a) and in the rolled $\mathrm{Cu}-\mathrm{Ag}$ (Fig. 4a), which lie within $30^{\circ}-35^{\circ}$; and that in a rolled $\alpha$-brass with laminar structure [51]. This difference may indicate that the mechanism that formed the localized band in the ARB material is not the same as those responsible for shear bands in these other materials.

To further examine the microstructure within the band, the band in Fig. 7c was thinned for TEM investigation. Figure 8a shows a section of the band as indicated by the parallel green lines. Layers across the band boundary bend about $7^{\circ}$ as demonstrated by the dashed red lines along two $\mathrm{Cu}-\mathrm{Nb}$ interfaces. The layers in the band have also become thinner and hence have plastically deformed. Interestingly comparing the electron diffraction patterns within the asprepared material (Fig. 8b) and the band (Fig. 8d), we find that the crystals have not rotated with the band. Within the band, the orientations of the $(111)_{\mathrm{Cu}}$ and $(10 \overline{1})_{\mathrm{Nb}}$ planes $\theta_{\mathrm{cu}}$ and $\theta_{\mathrm{Nb}}$ with respect to the fixed $x$-axis are distributed within $\left(18^{\circ}-27^{\circ}\right)$ and $\left(23^{\circ}-38^{\circ}\right)$ respectively. These ranges are very similar with those found in the as-prepared material, i.e., $\left(16^{\circ}-24^{\circ}\right)$ and $\left(24^{\circ}-35^{\circ}\right)$. The misorientation across the interface is also preserved. Further, the band angle $\beta$ of $\sim 25^{\circ}$ lies within the ranges of $\theta_{\mathrm{cu}}$ and $\theta_{\mathrm{Nb}}$ for the pair of $(111)_{\mathrm{Cu}}-(10 \overline{1})_{\mathrm{Nb}}$ slip planes, the most well-aligned for slip transmission across the interface. Taken together, the above findings suggest that this plastic instability occurred via dislocation slip transmitting across the interfaces on the well-aligned $(111)_{\mathrm{Cu}}-(10 \overline{1})_{\mathrm{Nb}}$ slip planes. Consequently this band could conventionally be termed a slip band rather than a shear band.

Figure 8c provides some evidence of slip transmission in association with formation of this trans-interface slip band. Several perfect dislocations approaching the interface could be identified as $1 / 2[01 \overline{1}]_{\mathrm{Cu}}$ or $1 / 2[10 \overline{1}]_{\mathrm{Cu}}$ which is confirmed by a Burgers circuit in the inset in Fig. 8c. Using the geometric relationship and the fixed coordinate system in Fig. 5f, interface transmission between dislocations with a Burgers vector of the $1 / 2[01 \overline{1}]_{\mathrm{Cu}}$ and $1 / 2[1 \overline{1} 1]_{\mathrm{Nb}}$ could be described by:

$$
1 / 2[01 \overline{1}]_{\mathrm{Cu}} \rightarrow \frac{1}{2}[1 \overline{1} 1]_{\mathrm{Nb}}+\mathrm{b}_{\mathrm{R}}
$$

where $b_{R}$ is the residual left behind after the transmission event, $1 / 2[0 \overline{1} 1]_{\mathrm{Cu}}=[-1.276,1.914$, $1.105] \AA, 1 / 2[1 \overline{1} 1]_{\mathrm{Nb}}=[-0.954,2.531,-0.918] \AA$, and thus $b_{R}=[-0.332,-0.617,-0.187] \AA$. The residual stored in the interface is relatively small compared to that which would be left if transmission were not permitted. 
We can conclude from the analysis of the $\mathrm{Cu}-\mathrm{Nb}$ system that as in the $\mathrm{Cu}-\mathrm{Ag}$ system, the transmissibility of the interface significantly impacts the type of plastic instability. The ARB interface, which permits slip transmission promotes nearly crystallographic slip bands. In contrast, resistance to slip interface transmission was associated with the shear bands found in the deformed PVD Cu-Nb pillar. It explains why the band angle and microstructures within the ARB slip band are different than those of the shear bands in the PVD $\mathrm{Cu}-\mathrm{Nb}$ and rolled $\mathrm{Cu}-\mathrm{Ag}$ composites. The interface that blocks impinging dislocations from transmitting responds by tilting crystallographically. The tilt can promote layer parallel slip, which is suspected to lead to shear banding.

\section{Conclusions}

In summary, using nanomechanical and bulk rolling in conjunction with TEM and HRTEM analysis, the mechanisms leading up to plastic instabilities in nanolayered composites are studied. We reveal that transmissibility of the interface plays a profound role on strain localization in these materials. Whether plasticity is mediated by slip or twinning, interface transmission leads to crystallographic band formation, whereas resistance to transmission leads to non-crystallographic band formation. We propose that an interface that blocks transmission can tilt the interface, as well as crystallographic orientations of the layers, and cause layer-parallel deformation. Layer parallel slip (or sliding) has previously been associated with shear banding in both conventional and nano-layered composites. The mechanistic insight provided in this work can enable use of interface engineering to enhance the ductility of nanolayered composites.

\section{Acknowledgements:}

This work is supported by the Center for Materials at Irradiation and Mechanical Extremes, an Energy Frontier Research Center funded by the U.S. Department of Energy, Office of Science, Office of Basic Energy Sciences under Award Number 2008LANL1026. This work was performed, in part, at the Center for Integrated Nanotechnologies, an Office of Science User Facility operated for the U.S. Department of Energy (DOE) Office of Science. Los Alamos National Laboratory, an affirmative action equal opportunity employer, is operated by Los Alamos National Security, LLC, for the National Nuclear Security Administration of the U.S. Department of Energy under contract DE-AC52-06NA25396. The authors thank Dr. Shuai Shao, Dr. Youxing Chen, and Prof. John P. Hirth for helpful discussions. 
References

[1] Misra A. 7 - Mechanical behavior of metallic nanolaminates. In: Hannink RHJ, Hill AJ, editors. Nanostructure Control of Materials. Woodhead Publishing, 2006. p.146.

[2] Zheng SJ, Carpenter JS, McCabe RJ, Beyehein IJ, Mara NA. Scientific Reports 2014;4:4226.

[3] Zheng SJ, Beyerlein IJ, Carpenter JS, Kang K, Wang J, Han WZ, A. MN. Nat Commun 2013;4:1696.

[4] Han W, Demkowicz MJ, Mara NA, Fu E, Sinha S, Rollett AD, Wang Y, Carpenter JS, Beyerlein IJ, Misra A. Adv. Mater. 2013;25:6975.

[5] Han WZ, Cerreta EK, Mara NA, Beyerlein IJ, Carpenter JS, Zheng SJ, Trujillo CP, Dickerson PO, Misra A. Acta Materialia 2014;63:150.

[6] Beyerlein IJ, Caro A, Demkowicz MJ, Mara NA, Misra A, Uberuaga BP. Mater. Today 2013;16:443.

[7] Mara NA, Bhattacharyya D, Hirth JP, Dickerson P, Misra A. Applied Physics Letters 2010;97:021909.

[8] Beyerlein IJ, Mara NA, Carpenter JS, Nizolek T, Mook WM, Wynn TA, McCabe RJ, Mayeur JR, Kang K, Zheng SJ, Wang J, Pollock TM. Journal of Materials Research 2013;28:1799.

[9] Tian YZ, Zhang ZF. Scripta Materialia 2013;68:542.

[10] Yan JW, Zhu XF, Yang B, Zhang GP. Physical Review Letters 2013;110.

[11] Lee SB, LeDonne JE, Lim SCV, Beyerlein IJ, Rollett AD. Acta Materialia 2012;60:1747.

[12] Yu-Zhang K, Embury JD, Han K, Misra A. Philosophical Magazine 2008;88:2559.

[13] Wang J, Misra A. Current Opinion in Solid State and Materials Science 2011;15:20.

[14] Paul H, Morawiec A, Driver JH, Bouzy E. International Journal of Plasticity 2009;25:1588.

[15] Tvergaard V, Needleman A. Proceedings of the Royal Society of London Series a-

Mathematical Physical and Engineering Sciences 1993;443:547.

[16] Steif PS. Journal of Applied Mechanics-Transactions of the Asme 1987;54:141.

[17] Steif PS. International Journal of Solids and Structures 1986;22:195.

[18] Anand L, Spitzig WA. Journal of the Mechanics and Physics of Solids 1980;28:113.

[19] Hirth JP. Metallurgical Transactions 1972;3:3047.

[20] Beyerlein I, Mara N, Wang J, Carpenter J, Zheng S, Han W, Zhang R, Kang K, Nizolek T, Pollock T. JOM 2012;64:1192.

[21] Beyerlein IJ, Wang J, Zhang R. APL Materials 2013;1.

[22] Shen TD, Schwarz RB, Zhang X. Applied Physics Letters 2005;87.

[23] Carpenter JS, Vogel SC, LeDonne JE, Hammon DL, Beyerlein IJ, Mara NA. Acta Materialia 2012;60:1576.

[24] Hirth JP, Lothe J. Theory of Dislocations. New York: Willey, 1982.

[25] Zhu YT, Liao XZ, Wu XL. Progress in Materials Science 2012;57:1.

[26] Beyerlein IJ, Toth LS, Tome CN, Suwas S. Philosophical Magazine 2007;87:885.

[27] Blewitt TH, Coltman RR, Redman JK. Journal of Applied Physics 1957;28:651.

[28] Cao F, Beyerlein IJ, Addessio FL, Sencer BH, Trujillo CP, Cerreta EK, Gray GT, III. Acta Materialia 2010;58:549.

[29] Yue YH, Liu P, Deng QS, Ma E, Zhang Z, Han XD. Nano Letters 2012;12:4045.

[30] Zheng SJ, Beyerlein IJ, Wang J, Carpenter JS, Han WZ, Mara NA. Acta Materialia 2012;60:5858.

[31] Beyerlein IJ, Mayeur JR, McCabe RJ, Zheng SJ, Carpenter JS, Mara NA. Acta Materialia 2014;72:137.

[32] McCabe RJ, Beyerlein IJ, Carpenter JS, Mara NA. Nat Commun 2014;5. 
[33] Beyerlein IJ, Mara NA, Bhattacharyya D, Alexander DJ, Necker CT. International Journal of Plasticity 2011;27:121.

[34] Tian YZ, Zhang ZF. Scripta Materialia 2012;66:65.

[35] Liu JB, Zeng YW, Meng L. Journal of Alloys and Compounds 2008;464:168.

[36] Wang YM, Sansoz F, LaGrange T, Ott RT, Marian J, Barbee TW, Hamza AV. Nature Materials 2013;12:697.

[37] Werner E, Prantl W. Acta Metall. Mater. 1990;38:533.

[38] Beyerlein IJ, Wang J, Kang K, Zheng SJ, Mara NA. Materials Research Letters 2013;1:89.

[39] Wang J, Zhou C, Beyerlein IJ, Shao S. JOM 2014;66:102.

[40] Wang J, Beyerlein IJ, Mara NA, Bhattacharyya D. Scripta Materialia 2011;64:1083.

[41] Han WZ, Carpenter JS, Wang J, Beyerlein IJ, Mara NA. Applied Physics Letters

2012;100:011911.

[42] Misra A, Hirth JP, Hoagland RG, Embury JD, Kung H. Acta Materialia 2004;52:2387.

[43] Carpenter JS, McCabe RJ, Beyerlein IJ, Wynn TA, Mara NA. Journal of Applied Physics 2013;113:094304.

[44] Wang J, Hoagland RG, Hirth JP, Misra A. Acta Materialia 2008;56:5685.

[45] Lehoczky SL. Physical Review Letters 1978;41:1814.

[46] Misra A, Hirth JP, Hoagland RG. Acta Materialia 2005;53:4817.

[47] Monclús MA, Zheng SJ, Mayeur JR, Beyerlein IJ, Mara NA, Polcar T, Llorca J, MolinaAldareguía JM. APL Materials 2013;1:052103.

[48] Demkowicz MJ, Thilly L. Acta Materialia 2011;59:7744.

[49] Wang J, Kang K, Zhang R, Zheng S, Beyerlein I, Mara N. JOM Journal of the Minerals, Metals and Materials Society 2012;64:1208.

[50] Zhang RF, Germann TC, Wang J, Liu XY, Beyerlein IJ. Scripta Materialia 2013;68:114.

[51] Yeung WY, Duggan BJ. Acta Metallurgica 1987;35:541. 
Captions:

Figure 1: (a) a bright field TEM image showing the colonies containing $\mathrm{Cu}-\mathrm{Ag}$ nanolamellae in the as-cast material; (b) a magnified TEM image of the nanolayered $\mathrm{Cu}-\mathrm{Ag}$ composite and corresponding electron diffraction patterns. The diffraction patterns can be indexed as $\langle 110\rangle \mathrm{Cu}$ marked with white and red rectangles and $\langle 110\rangle \mathrm{Ag}$ marked with yellow and grain rectangles. According to the diffraction patterns, $\mathrm{Cu}-\mathrm{Ag}$ interface can be identified as $(111) \mathrm{Cu}-(111) \mathrm{Nb}$, and $\mathrm{Cu}-\mathrm{Ag}$ orientation relationships are cube-on-cube suggested by the white-yellow or red-green patterns and hetero-twin indicated by the white-green or red-yellow patterns.

Figure 2: HRTEM images of a typical cube-on-cube $\mathrm{Cu}-\mathrm{Ag}$ interface (a) and a typical hetero-twin $\mathrm{Cu}$ $\mathrm{Ag}$ interface (b), which contains similar misfit dislocations and atomic steps indicated by red arrows; (c) a schematic of the cube-on-cube interface; (d) a schematic of the hetero-twin interface.

Figure 3: (a) a TEM image showing that twins in an Ag layer can transmit across the interface and facilitate twinning in an adjacent $\mathrm{Cu}$ layer; (b)-(c) evidence of twin transmission across the interface; (d) a schematic of the final interface structure after twin transmission.

Figure 4: (a) a shear band in the $\mathrm{Cu}-\mathrm{Ag}$ composite where hetero-twin interfaces prevail; (b) a TEM image taken close to the shear band in (a) showing the interface tilting; (c) a HRTEM image of the interface in (b); (d)-(e) schematic diagrams of interface tilting resulting from Ag twinning.

Fig. 5: typical TEM images of the as-deposited PVD (a) and the as-fabricated ARB (b) $\mathrm{Cu}-\mathrm{Nb}$ nanocomposites; HRTEM images of the atomic flat PVD (c) and faceted ARB (d) Cu-Nb interfaces showing the aligned slip planes indicated by green lines and misfit dislocations; schematic diagrams of the PVD (e) and ARB (f) $\mathrm{Cu}-\mathrm{Nb}$ interfaces.

Figure 6: (a) a shear band in the PVD Cu-Nb multilayers; (b) an illustration of the dislocationinterface interaction; (c) interface rotation resulting from the dislocation-interface interaction, which can activate slip systems parallel to the interface and lead to shear band finally.

Figure 7: (a) a SEM image of the pillar before the compression, (b) a SEM image of the pillar after the compression, in which the localized deformation is indicated by an arrow, (c) a SEM image of the deformed micropillar cross-sectioned by FIB showing a band with an angle of about $25^{\circ}$.

Figure 8: (a) a TEM image of the slip band exhibiting layer bending across the shear band and twins nearby the shear band; (c) a HRTEM image explaining full dislocation transmission. The inset is an enlarged $\mathrm{Cu}$ full dislocation identified by a Burgers circuit; (b) electron diffraction pattern corresponding to the as-prepared material; (d) electron diffraction pattern corresponding to the slip 
band region in the compressed pillar. It is found that after the shear banding the orientation distribution of both $\mathrm{Cu}$ and $\mathrm{Nb}$ has no significant change. 

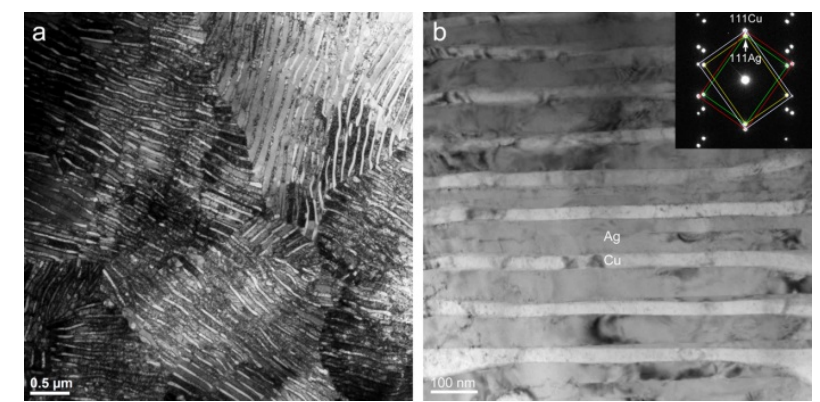

Figure 1
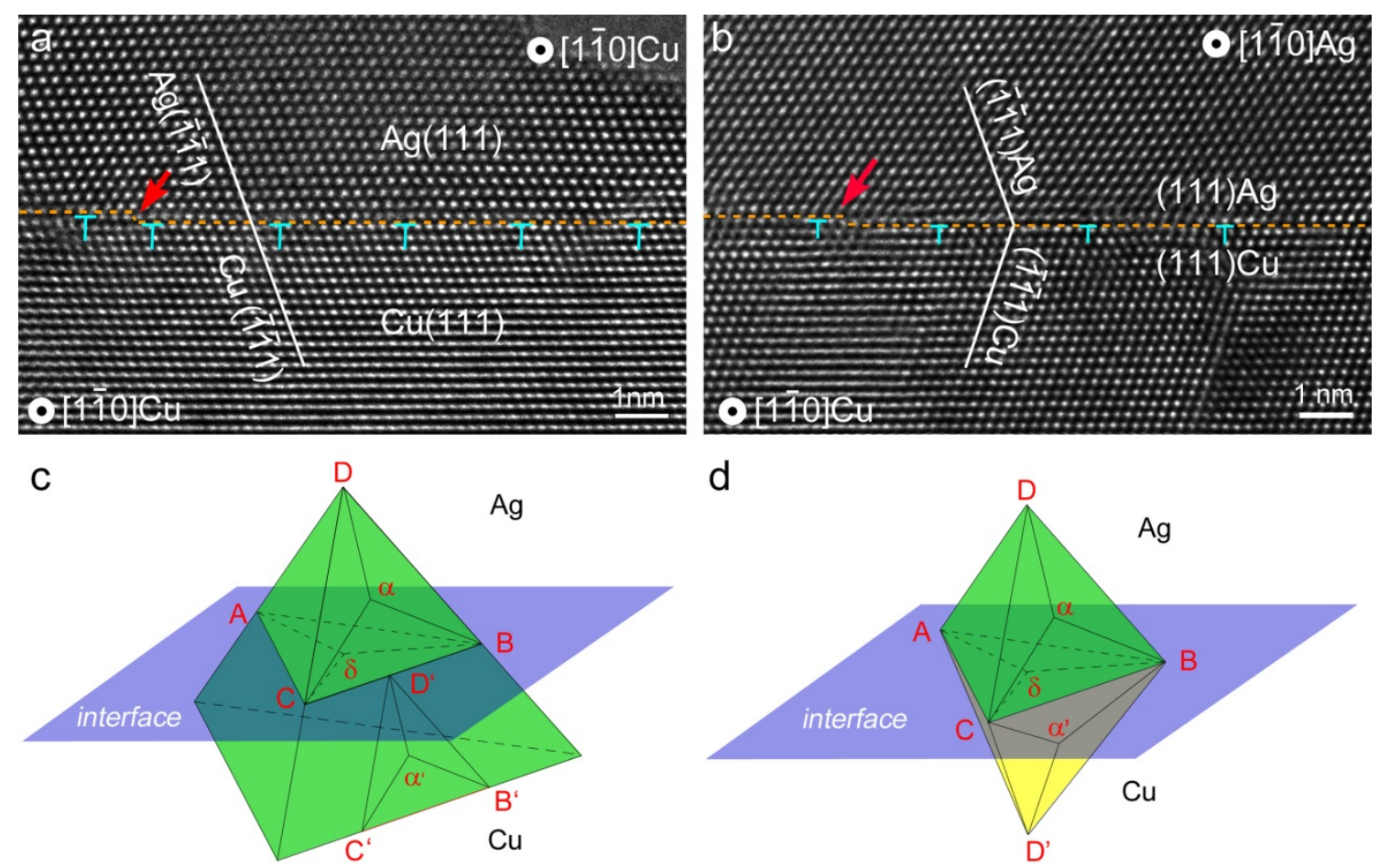

Figure 2 

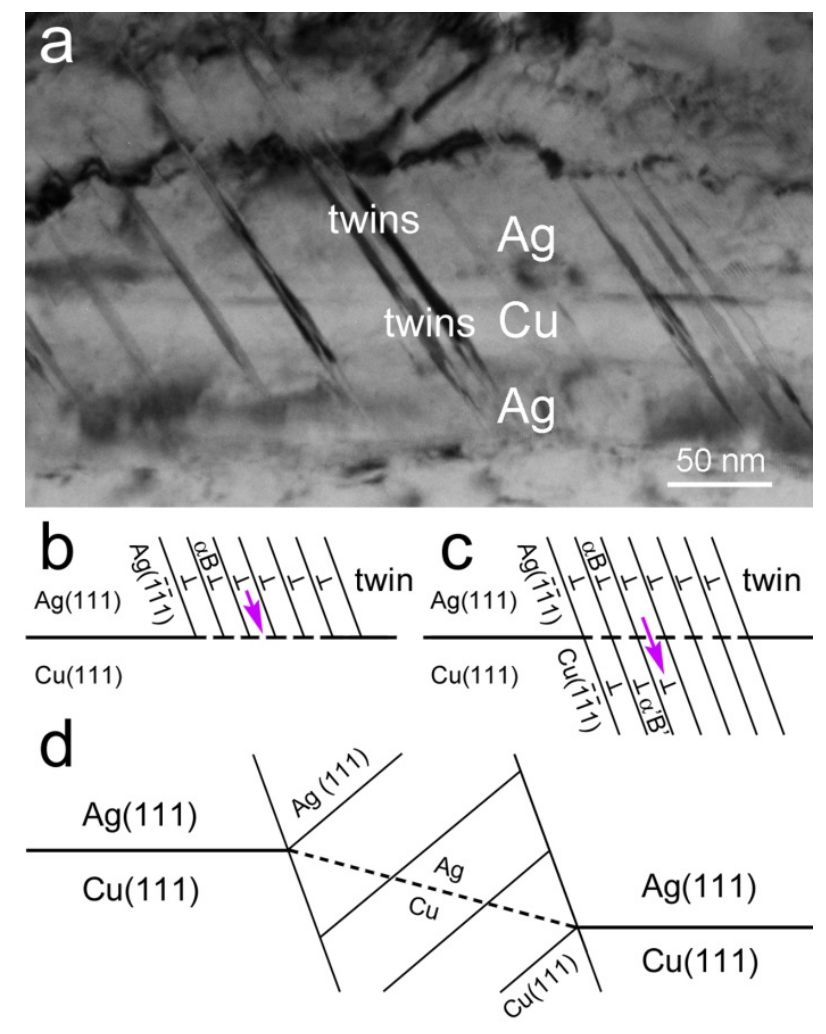

Figure 3
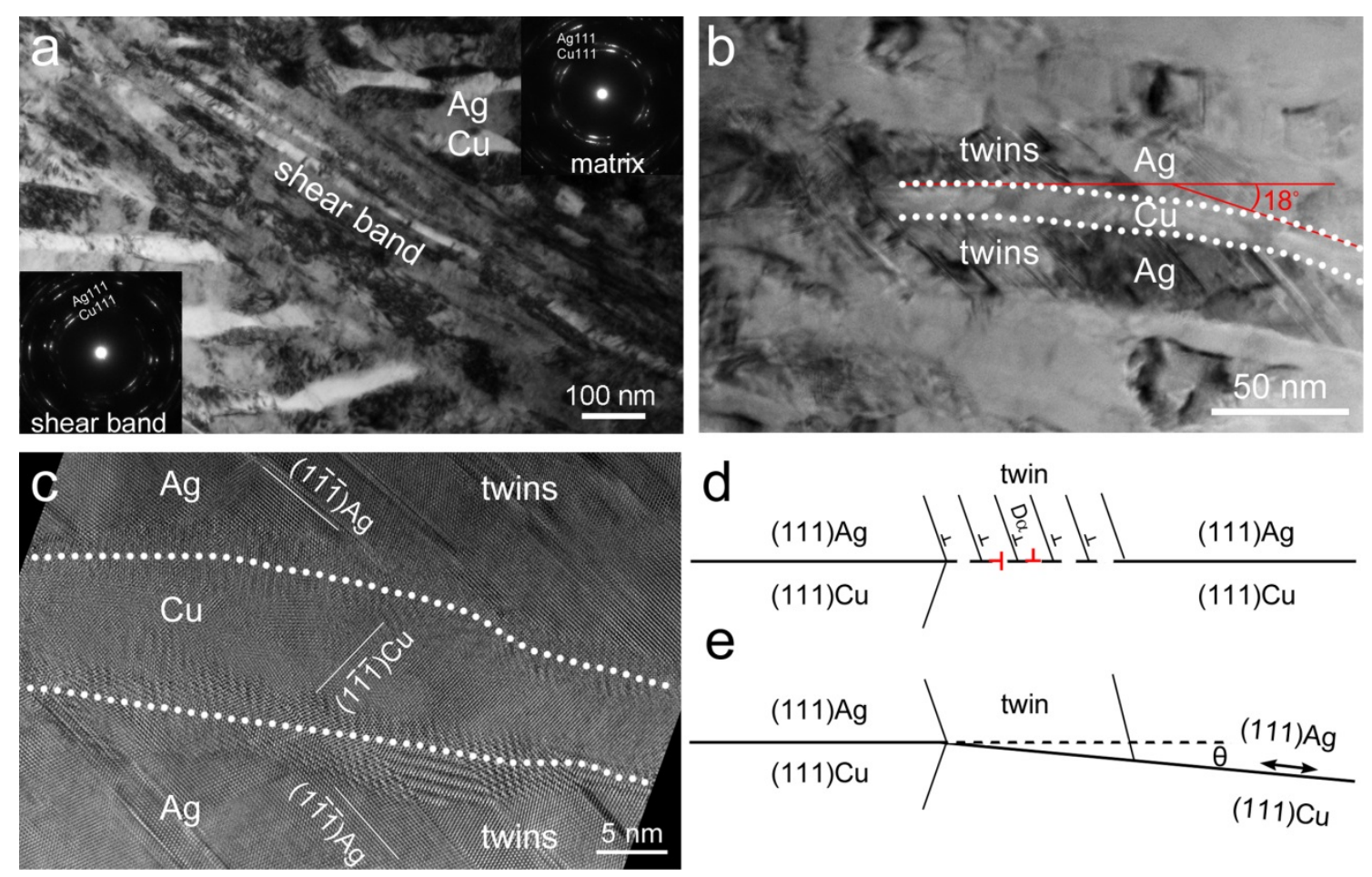

Figure 4 

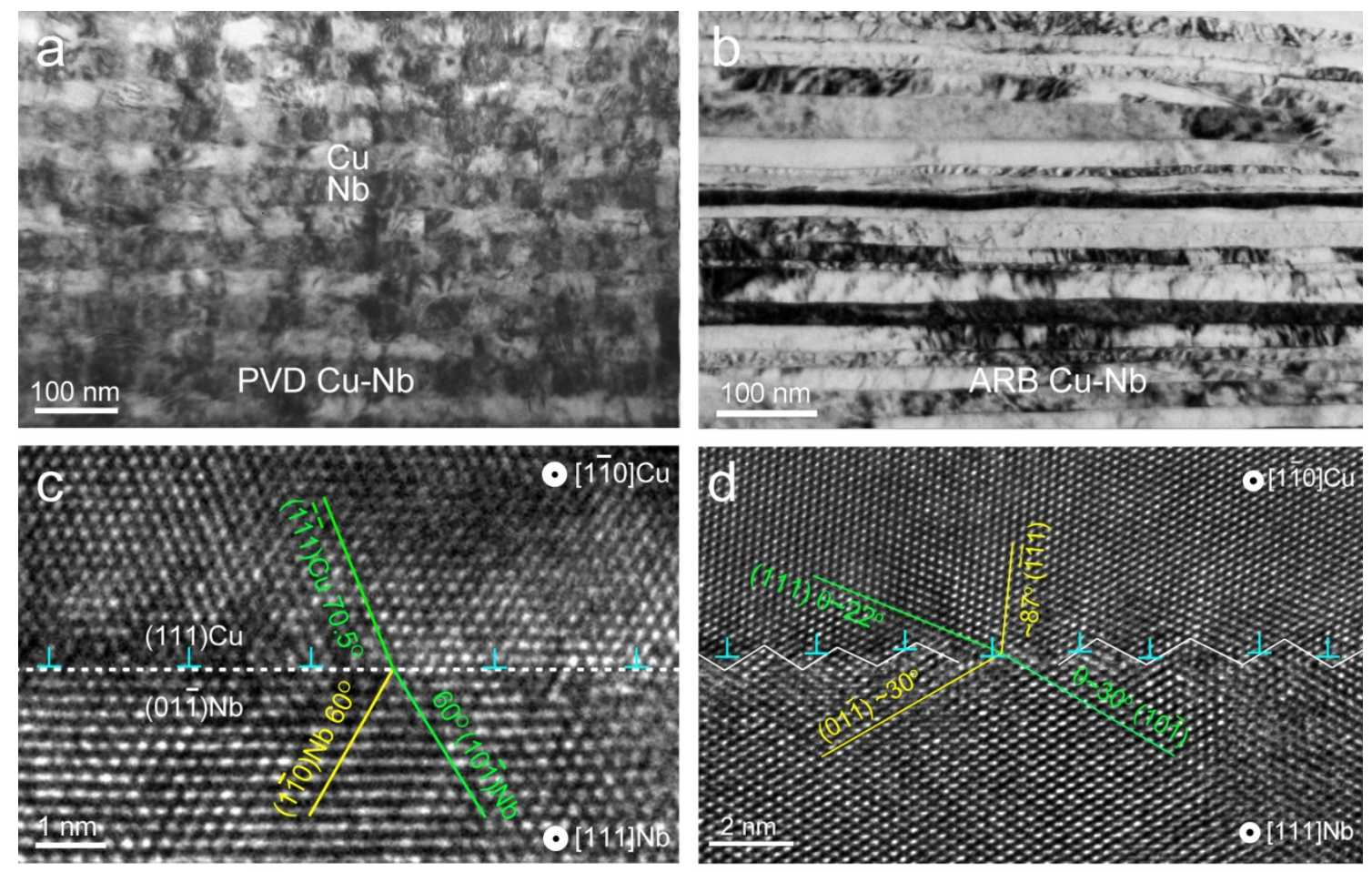

e

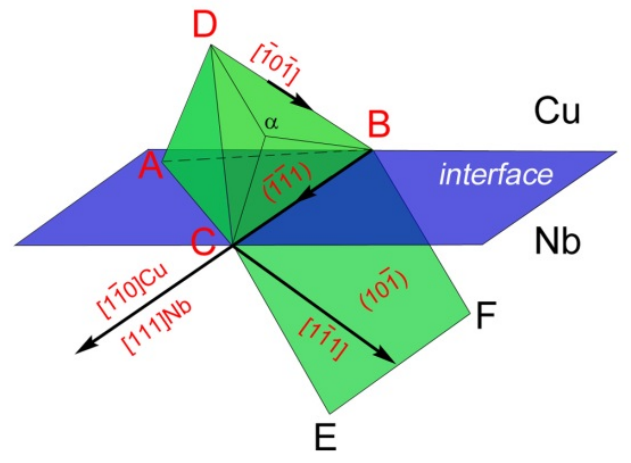

f

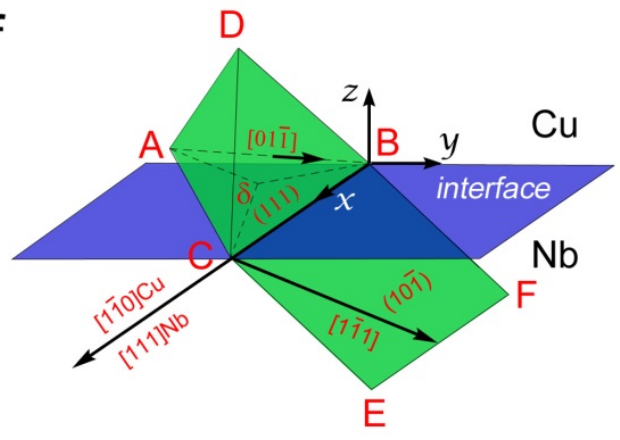

Figure 5 

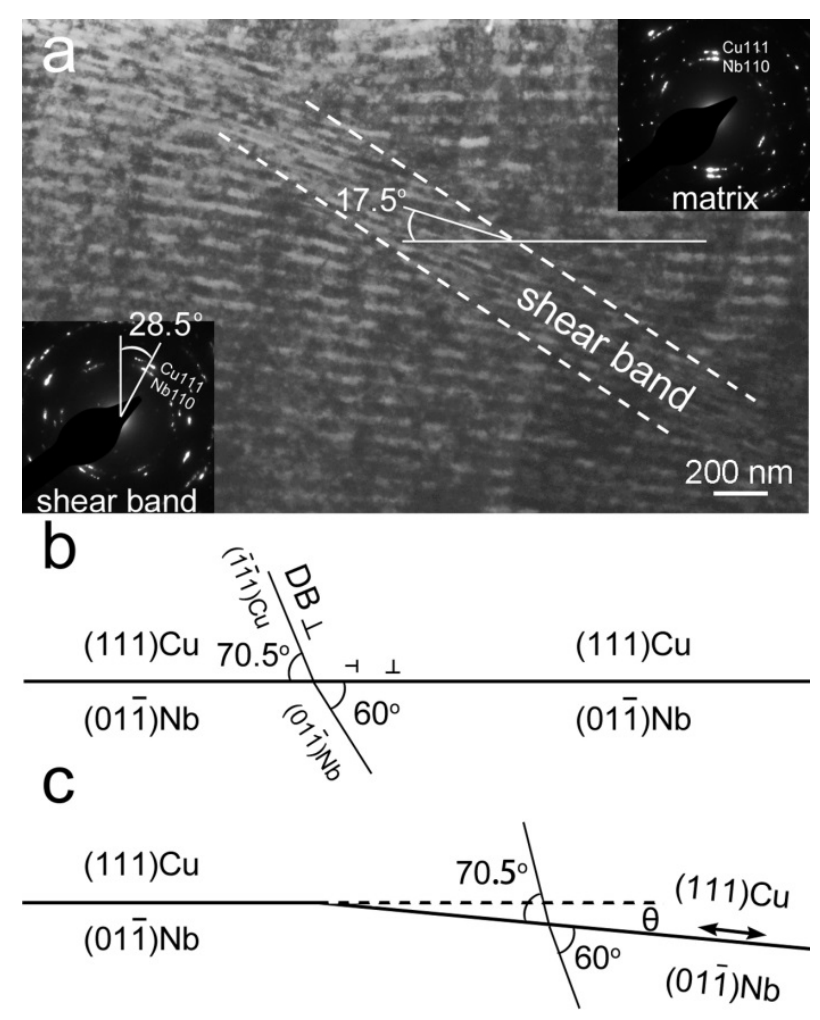

Figure 6
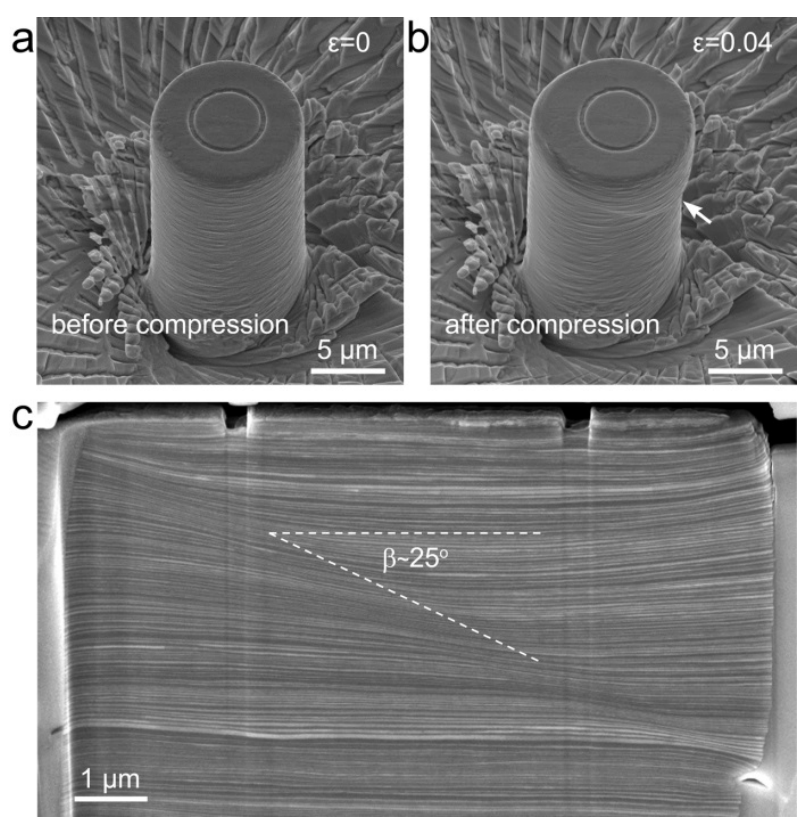

Figure 7 

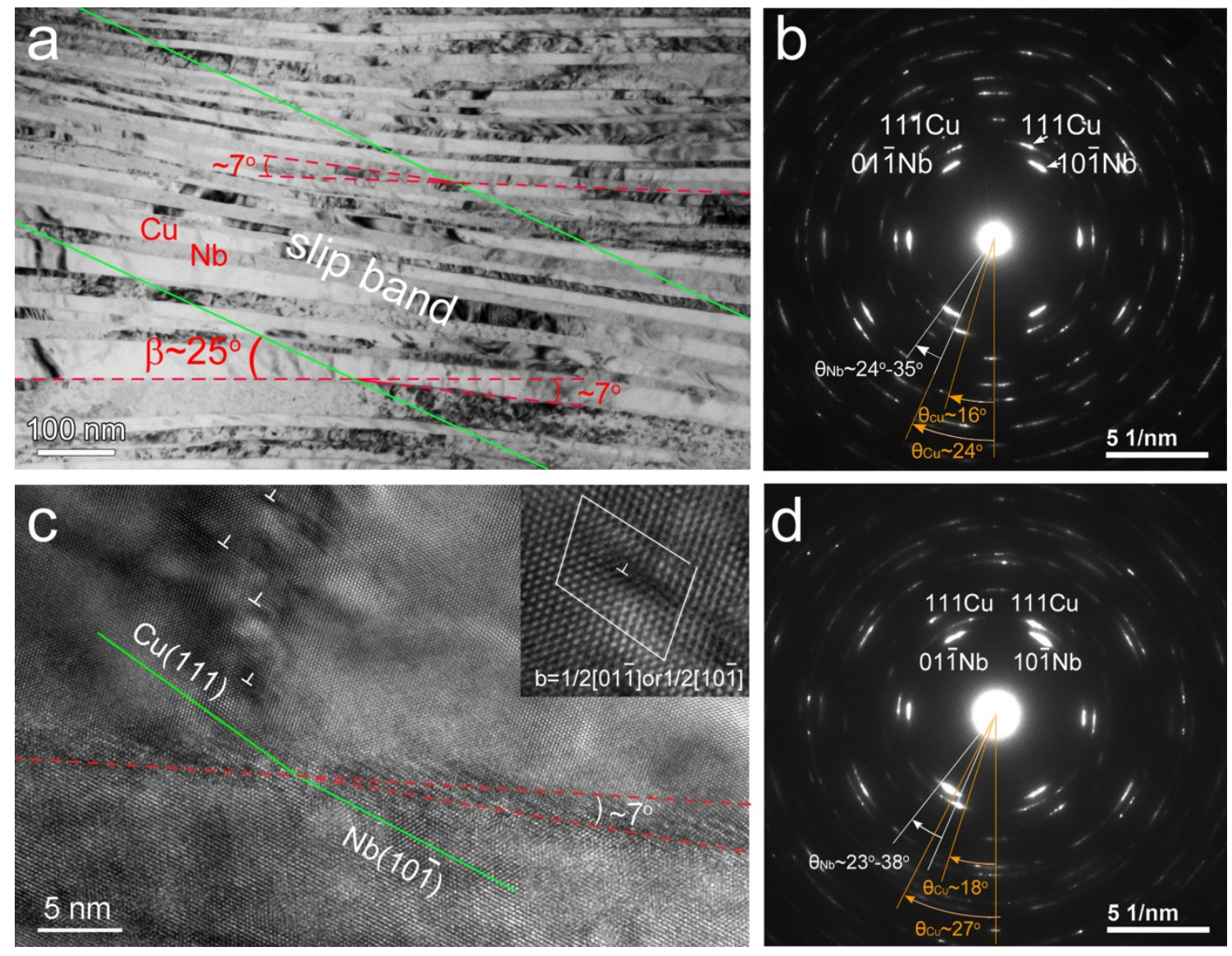

Figure 8 